\title{
Surgery without preoperative histological confirmation of lung cancer: what is the current clinical practice?
}

\author{
Mohammad R. Ghamati ${ }^{1,2}$, Wilson W. L. Li ${ }^{1}$, Erik H. F. M. van der Heijden ${ }^{3}$, Ad F. T. M. Verhagen ${ }^{1}$, \\ Ronald A. Damhuis ${ }^{4}$
}

${ }^{1}$ Department of Cardiothoracic Surgery, Radboud University Medical Centre, Nijmegen, The Netherlands; ${ }^{2}$ Department of Cardiothoracic Surgery, Isala Heart Centre, Zwolle, The Netherlands; ${ }^{3}$ Department of Pulmonary Diseases, Radboud University Medical Centre, Nijmegen, The Netherlands; ${ }^{4}$ Department of Research, Netherlands Comprehensive Cancer Organization, Utrecht, The Netherlands

Contributions: (I) Conception and design: All authors; (II) Administrative support: MR Ghamati, WWL Li; (III) Provision of study materials or patients: MR Ghamati, WWL Li, RA Damhuis; (IV) Collection and assembly of data: MR Ghamati, WWL Li, RA Damhuis; (V) Data analysis and interpretation: All authors; (VI) Manuscript writing: All authors; (VII) Final approval of manuscript: All authors.

Correspondence to: Mohammad R. Ghamati. Department of Cardiothoracic Surgery, Radboud University Medical Centre, P.O. Box 9101, 6500 HB, Nijmegen, The Netherlands. Email: mohammad.ghamati@radboudumc.nl.

Background: There are discordances in the guidelines regarding the need to acquire histological diagnosis before surgical treatment of (presumed) lung cancer. Preoperative histological confirmation is always encouraged in this setting to prevent unnecessary surgery or when sublobar resection for small-sized tumors is considered. The aim of this retrospective cohort study was to assess the proportion of patients undergoing lung cancer resection in the Netherlands without preoperative pathological confirmation, based on the intraoperative pathological diagnosis (IOD) rate, and to determine characteristics that may influence IOD frequency.

Methods: Data on 10,226 patients, who underwent surgical treatment for lung cancer from 2010 to 2015 , were retrieved from the Netherlands National Cancer Registry. We registered an IOD when the date of diagnosis equaled the date of the first surgical intervention. Tabulations and multivariable logistic regression were used to identify predictive parameters for IOD.

Results: $36 \%$ of surgical procedures were classified as IOD, and decreased with increasing tumor size and extent of surgery ( $57 \%$ for segmentectomy, 39\% for lobectomy and $11 \%$ for pneumonectomy). IOD was more frequently observed in adenocarcinoma (41\%), varied between hospitals from $13 \%$ to $66 \%$ and was less common when patients were referred from a hospital where thoracic surgery was not performed. Previous history of cancer did not affect IOD.

Conclusions: More than one-third of patients with suspected lung cancer in the Netherlands was operated without preoperative histological confirmation. There was significant variation in IOD rates between different hospitals, which deserves further detailed analysis when striving for uniform surgical quality of care for patients with lung cancer.

Keywords: Intraoperative pathological diagnosis; preoperative histological confirmation; lung cancer surgery; lung cancer diagnosis

Submitted May 12, 2021. Accepted for publication Aug 13, 2021.

doi: $10.21037 /$ jtd-21-617

View this article at: https://dx.doi.org/10.21037/jtd-21-617 


\section{Introduction}

There is strong agreement amongst guidelines that surgical resection forms the cornerstone of curative treatment for patients with early-stage lung cancer (1-5). However, discordances exist regarding the need to acquire pathological diagnosis before planned surgical resection (Table 1). For example, the European Society for Medical Oncology (ESMO) guidelines on early nonsmall cell lung cancer (NSCLC) recommend to acquire pathological diagnosis before any curative treatment, unless an experienced multidisciplinary group decides the risks of obtaining pathology to be unacceptable (4). Conversely, the United States (US)-based National Comprehensive Cancer Network (NCCN) guidelines recommend that patients with a strong clinical suspicion of early-stage lung cancer do not require a biopsy before surgery (5); however, an intraoperative tissue diagnosis is still necessary before performing intended curative resection, usually through intraoperative frozen section (FS) procedure. Comparably, the American College of Chest Physicians (ACCP) and British Thoracic Society (BTS) have advocated upfront surgery when the clinical probability of malignancy is high, either $>65 \%$ (6) or $>70 \%$ (3).

Whether preoperative pathological confirmation of lung cancer diagnosis is indispensable is an interesting debate. Case series have reported risks of $5-20 \%$ of finding benign disease (thus ultimately undergoing unnecessary surgery) when patients were operated without preoperative pathological diagnosis (7-13). Moreover, with a rising trend of utilizing sublobar resections to treat smallsized early-stage lung cancer (14), accurate pathological classification beforehand will become increasingly important. After all, high-risk histological subtypes such as solid or micropapillary adenocarcinoma are associated with local recurrence in patients who have undergone limited resections when compared to lobectomy (15), and the diagnostic accuracy of intraoperative FS on these high-risk histologic features is still debatable (16).

With conflicting recommendations from available guidelines, it is reasonable to assume this will lead to variation in lung cancer surgical care. Recent studies have reported important quality gaps in treatment delivery (17) and unwarranted care variation (18), leading to significant variation in preoperative cardiopulmonary assessment practices (19) and surgical resection rates (20). Whether there are also differences in preoperative diagnostic testing, leading to variation in proportion of patients being operated without a preoperative pathological diagnosis, remains to be clarified.

In our current study, we assessed the proportion of patients undergoing lung cancer resection being operated in the Netherlands without preoperative pathological confirmation, based on the rate of "intraoperative pathological diagnosis" (IOD). Furthermore, we attempted to determine factors that are predictive for IOD, as well as variations in these rates in the Netherlands, with specific interests for patients with small-sized early-stage disease and undergoing sublobar resection. Our hypothesis is that IOD rate will be higher in small peripheral tumors that are resected by smaller anatomical resections. Mainly because of the difficulty to reach these tumors with current diagnostic tools. And we also can assume that conflicting recommendations in the guidelines will lead to a significant IOD rate variation in the Netherlands.

We present the following article in accordance with the STROBE reporting checklist (available at https://dx.doi. org/10.21037/jtd-21-617).

\section{Methods}

This study was designed as a retrospective cohort study, including all surgical treated patients in the Netherlands using data from a national wide database. The study was conducted in accordance with the Declaration of Helsinki (as revised in 2013).

Data used in this study was obtained from the Netherlands National Cancer Registry (NNCR), after approval by the NCCR Privacy Review Board. In accordance with the regulations of the Central Committee on Research involving Human Subjects, this type of study does not require approval from an ethics committee in the Netherlands.

All registered patients in the NNCR with invasive primary lung cancer who underwent an anatomical resection, diagnosed between 2010 and 2015 were evaluated for inclusion in this study.

The NNCR collects data on all cancer patients diagnosed in the Netherlands, based on notification of newly diagnosed malignancies by the national automated pathology archive and of hospital discharge diagnoses. Information on demographics, diagnosis, staging, and treatment is extracted routinely from the medical records by specially trained NNCR personnel and not by the care takers themselves, in order to minimalize the chance of bias. Clinical and postsurgical stage information was recorded according to the 7th edition of the TNM Classification of Malignant Tumours from the International Union against 
Table 1 Guidelines recommendations on the need of preoperative diagnosis prior to surgical resection

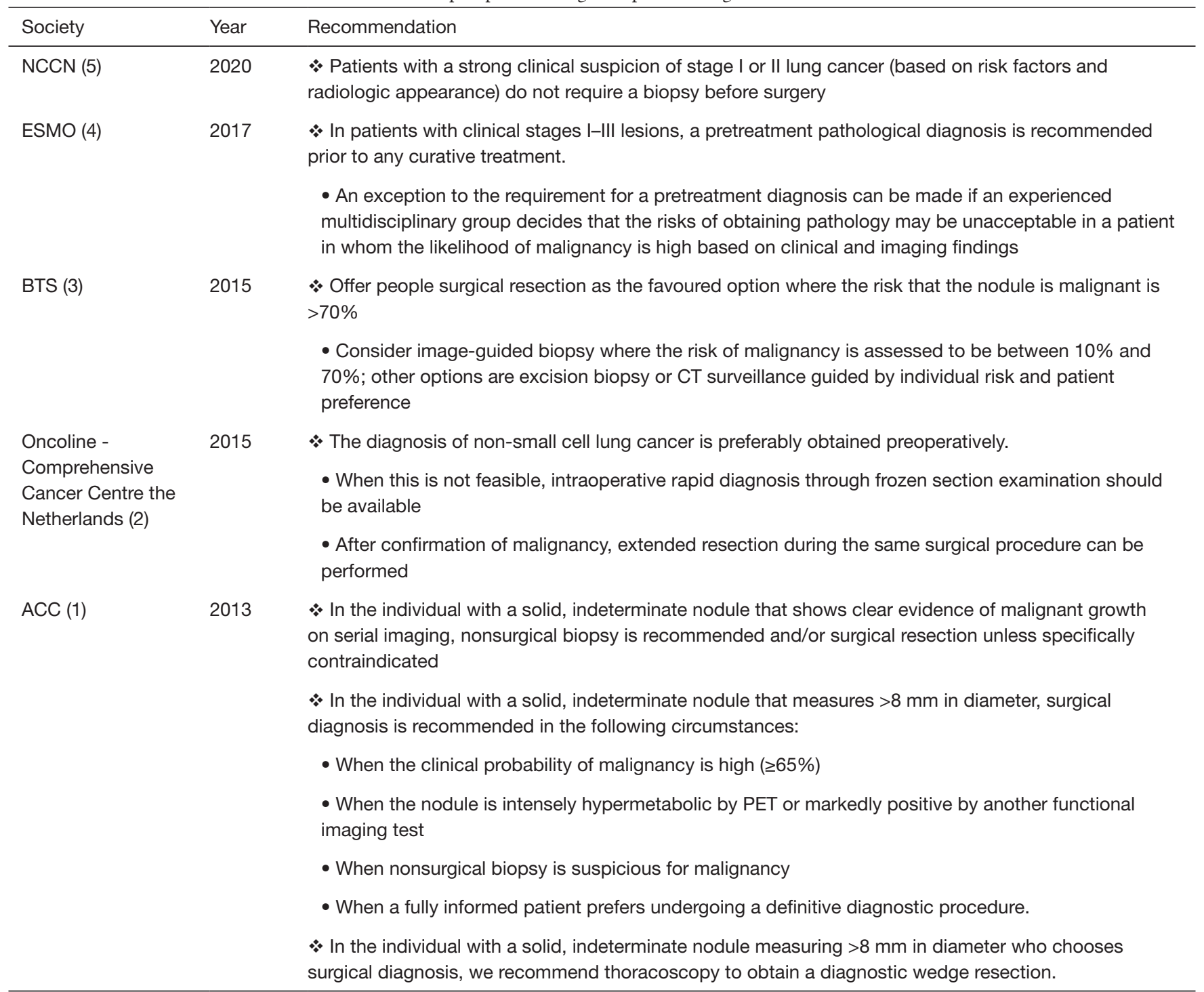

NCCN, national comprehensive cancer network; ESMO, European society for medical oncology; BTS, british thoracic society; ACC, American college of chest physicians.

Cancer. The date of diagnosis is recorded as the date of retrieval of cytology specimens or histological specimen, mentioned in the pathology report that first confirmed the diagnosis of cancer. Whether diagnosis resulted from FS analysis during surgery or by later examination of the resection specimen, cannot be distinguished. Lung surgery for benign lesions is not recorded. Hospitals of diagnosis and treatment are recorded separately.

In 2010, 92 hospitals treated patients with lung cancer, while 58 hospitals performed lung surgery. Fifteen of the
58 hospitals stopped performing lung surgery during the study period. Patients who were younger than 18 years of age, or with cancer located in the main bronchi, operated abroad or in any of the hospitals that discontinued lung surgery, were excluded from this study. Patients with synchronous or metachronous tumors were excluded in order to keep a homogeneous population. Patients who underwent wedge resection were also excluded because in the NNCR database it was not recorded whether the procedure was performed with diagnostic or with curative intend. 


\section{Study variables}

Age was categorized in 4 subgroups. Pathology was coded according to the ICD-O-3 morphology classification and was categorized in 5 subgroups: small cell lung cancer, squamous cancer, adenocarcinoma, large cell carcinoma not otherwise specified (NOS), and other tumors including neuroendocrine tumors, carcinoids, and sarcomatoid lesions. Type of surgery was categorized into segmentectomy, lobectomy, bilobectomy, and pneumonectomy. IOD was designated when the date of diagnosis equaled the date of the first operation. IOD could be achieved by an intraoperative frozen section or by definitive pathological examination after the surgery. This could not be distinguished because the data regarding a frozen section with pathological examination or a intraoperative cytological examination is not recorded in the NNCR. History of previous cancer was included as a parameter, excluding diagnoses of non-melanoma skin cancer. A separate parameter designates that diagnosis and surgery did not occur in the same hospital.

\section{Statistical analysis}

Statistical analyses were performed using Stata 14 (CCopyright 1996-2017 StataCorp LLC). The proportion of patients with intraoperative diagnosis was tabulated, this was also done for the sub analysis of patients undergoing sublobar resection. Missing data were included as separate categories. Differences between subgroups were tested for significance with chi-square statistics. Multivariable analysis was performed using hierarchical logistic regression, including the hospital of surgery as the first level variable.
The hierarchical model accounts for clustering of patients within hospitals and allows assessment of residual variation in IOD rate between hospitals.

The significance of predictive factors was assessed with the log-likelihood test and significant $(\mathrm{P}<0.05)$ parameters are represented by odds ratios (OR) and a $95 \%$ confidence interval (CI). Posterior Bayes estimates of the odds ratios for the involved hospitals were plotted against hospital resection volume. The hospital resection volume is defined as the number of anatomical parenchymal resections performed for lung cancer in a hospital.

\section{Results}

A total of 10,226 patients in 43 hospitals were included in our analysis (Table 2). The median age was 66 years and $57 \%$ were men. Adenocarcinoma was the most common pathological subtype $(52 \%)$ and small cell lung cancer comprised only $1,4 \%$ of cases. Lobectomy comprised $83 \%$ of all operations and segmentectomy was only performed in $1.7 \%$ of cases. Clinical T-stages $1 \mathrm{a}(0-2 \mathrm{~cm})$ and $1 \mathrm{~b}$ $(2-3 \mathrm{~cm})$ were diagnosed in $24 \%$ and $17 \%$ respectively. Of all patients, $16 \%$ were previously diagnosed with another type of cancer and $25 \%$ were transferred from the hospital of diagnosis to another hospital for lung cancer surgery.

\section{Intraoperative pathological diagnosis}

Cytological or histological diagnosis was not available before the start of surgery for more than one-third of the patients (36\%) (Table 2). This proportion remained stable over time from $38 \%$ in $2010-2011$ to $35 \%$ in $2014-2015$, and was not affected by age or sex. The IOD proportion decreased with

Table 2 Proportion of IOD in patients who underwent lung cancer surgery

\begin{tabular}{|c|c|c|c|c|c|}
\hline & Cohort, $N=10,226$ & $\mathrm{~N}$ [\%] with IOD & $P$ value & OR & $95 \% \mathrm{Cl}$ \\
\hline \multicolumn{6}{|l|}{ Age } \\
\hline $18-59$ & 2,638 & 923 [35] & 0.39 & NS & - \\
\hline $60-69$ & 3,992 & $1,437[36]$ & & & \\
\hline $70-79$ & 3,171 & $1,142[36]$ & & & \\
\hline $80+$ & 425 & $140[33]$ & & & \\
\hline \multicolumn{6}{|l|}{ Gender } \\
\hline Men & 5,831 & 1,924 [33] & $<0.001$ & NS & - \\
\hline Women & 4,395 & 1,714 [39] & & & \\
\hline
\end{tabular}

Table 2 (continued) 
Table 2 (continued)

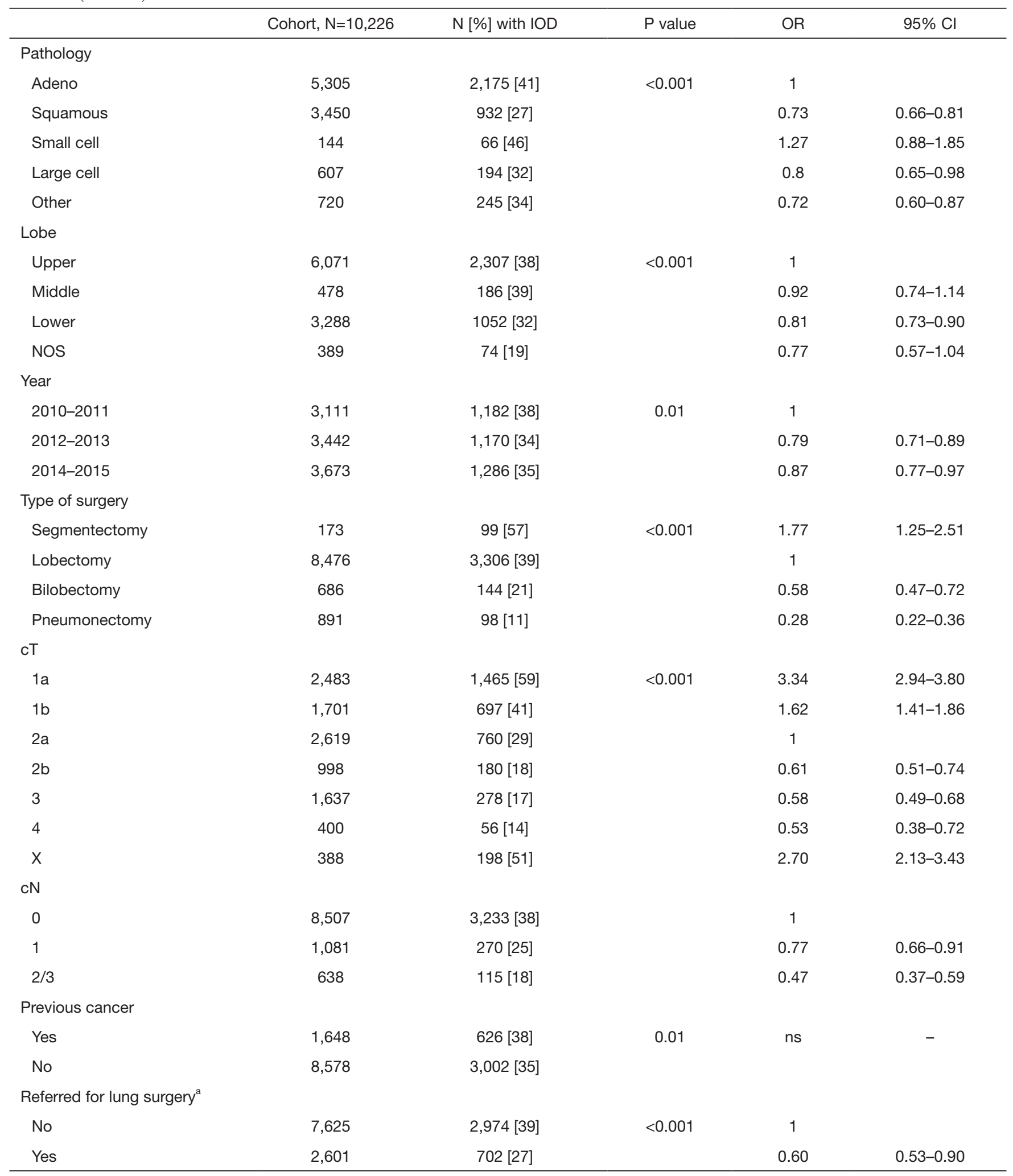

a patients were analyzed in another hospital than the hospital of surgery. IOD, intraoperative pathological diagnosis; OR, odds ratio; Cl, confidence Interval; NS, not significant; NOS, not specified/missing; cT, clinical T stage; cN, clinical N stage; X, missing/unknown. 


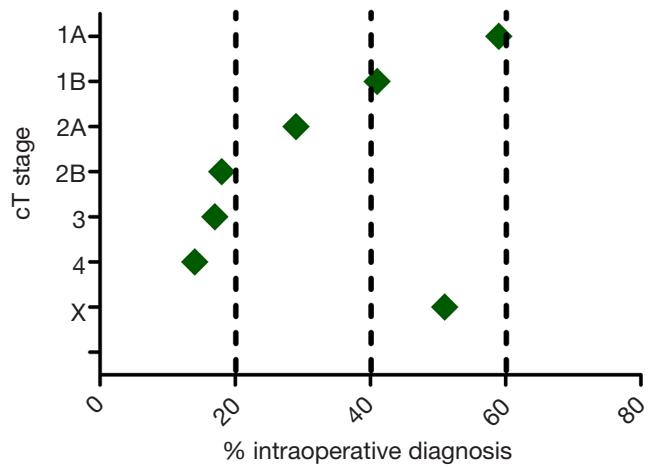

Figure 1 Proportion of IOD in relation to the clinical T (cT) stage. IOD, intraoperative pathological diagnosis; $\mathrm{c} T$, clinical $\mathrm{T}$.

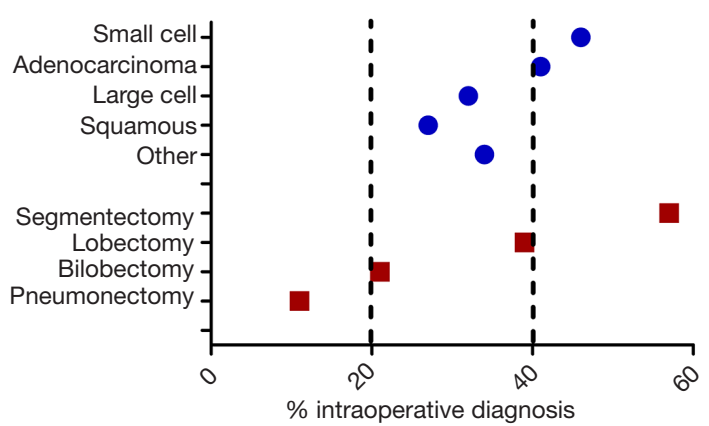

Figure 2 Proportion of IOD in relation to the histological cancer type (blue circles) and the extent of resection (red squares). IOD, intraoperative pathological diagnosis.

tumor size, being $59 \%$ for $\mathrm{T} 1 \mathrm{a}, 41 \%$ for $\mathrm{T} 1 \mathrm{~b}$, and only $17 \%$ and $14 \%$ for T3 and T4 tumors respectively (Figure 1). Regarding differences between pathological subtypes, IOD was highest (46\%) for small cell carcinoma; the frequency of IOD was more common in adenocarcinoma (41\%) than in other non-small cell lung cancer subtypes (Figure 2). IOD varied significantly with different extent of surgical resection, being $57 \%$ for segmentectomy, 39\% for lobectomy, and $11 \%$ for pneumonectomy (Figure 2). A history of previous cancer had no influence on the IOD proportion, but the IOD proportion was significantly lower if patients were not diagnosed in the hospital of surgery ( $27 \%$ versus $39 \%, \mathrm{P}<0.001)$.

A small group of patients $(\mathrm{N}=173)$ underwent a segmentectomy. Sub-analysis showed that adenocarcinoma was the most common pathological diagnosis in this group $(65 \%)$ with an IOD rate of $57 \%$ (Table 3). Squamous cell carcinoma was diagnosed in $14 \%$ with a high IOD rate of
$72 \%$. A total of 18 patients (10\%) had a tumor classified T2b or higher, with a IOD rate of $44 \%$ in this group. In $9 \%(\mathrm{~N}=16)$ of the cases the $\mathrm{N}$ stage was 1 or higher with an IOD rate of $31 \%$.

Multivariable analysis revealed the independent predictive impact of pathology, tumor localization, year of diagnosis, type of surgery, TNM stage, and patient referral (Table 2). Controlling for the independent prognostic factors, residual variation in IOD rate between hospitals was considerable $(\mathrm{P}<0.001)$. Odds ratios ranged from 0.23 to 3.98 , reflecting proportions of IOD ranging from $13 \%$ to $66 \%$. IOD proportions were significantly higher in 18 hospitals and lower in 14 hospitals, compared to the national average (Figure 3).

\section{Discussion}

Our study has shown that over one-third of patients who had undergone lung cancer resection were operated without a preoperative definitive diagnosis in current Dutch clinical practice. IOD rate decreased with increasing tumor size and extent of surgery. Furthermore, there was significant variation in the rate of IOD, varying from $13-66 \%$ between the Dutch surgical centers during the study period.

Our findings are roughly comparable with the scarcely reported data on this matter. Population-based registries have documented rates of approximately $30 \%$ of cases without pathological confirmation of their lung cancer diagnosis, regardless of treatment strategy (21). For lung cancer patients undergoing operative treatment specifically, various small single-institutional studies reported that $27-36 \%$ of patients who had undergone surgery for (ultimately) confirmed lung cancer were operated without preoperative pathological diagnosis $(9,22)$. To the best of our knowledge, this is the first nationwide populationbased registry describing the rate of lung cancer patients undergoing surgical treatment without a preoperative definitive diagnosis.

The lack of a definite preoperative diagnosis, leading to IOD, generates various potential risks and problems during lung cancer surgery. First of all, there is an obvious possibility that a patient with a suspicious pulmonary nodule without definitive diagnosis might be operated for benign disease, thus resulting in an unnecessary surgical procedure and related morbidity and risks in hindsight. Due to our methodology, utilizing data from the NNCR where patients with benign diagnoses were already excluded, this risk cannot be estimated in our current study. However, the rate 
Table 3 Subanalysis of IOD rate in patients who underwent segmentectomy for lung cancer $(\mathrm{N}=173)$

\begin{tabular}{|c|c|c|}
\hline & $\mathrm{N}$ & $\mathrm{N}[\%]$ with IOD \\
\hline \multicolumn{3}{|l|}{ Pathology } \\
\hline Adenocarcinoma & 111 & $63[57]$ \\
\hline Small cell carcinoma & 3 & $1[33]$ \\
\hline large cell carcinoma & 7 & 2 [29] \\
\hline \multicolumn{3}{|l|}{ cT } \\
\hline $1 \mathrm{a}$ & 96 & $65[68]$ \\
\hline $1 b$ & 39 & $20[51]$ \\
\hline $2 a$ & 20 & 5 [25] \\
\hline $1 / 2 / 3$ & 16 & $5[31]$ \\
\hline
\end{tabular}

IOD, intraoperative pathological diagnosis; cT, clinical T stage; $\mathrm{cN}$, clinical $\mathrm{N}$ stage.

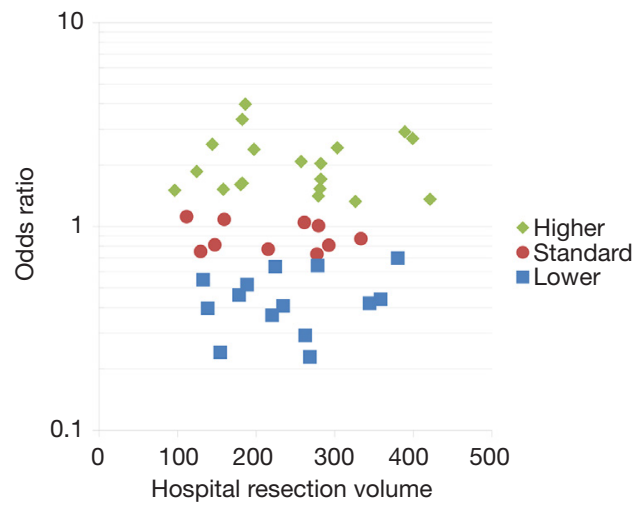

Figure 3 OR of the IOD proportion, in relation to hospital resection volume. Grouped in standard OR, higher and lower than the standard OR. IOD, intraoperative pathological diagnosis; OR, odds ratio.

of unnecessary surgery was not the focus of our analysis, and this (often trivialized) risk has already been reported in other investigations, varying from $5-20 \%$ in case series published in the last decade (7-13). For example, a recent study on 129 patients undergoing surgical intervention with pulmonary nodules found incidentally or by lung cancer screening (13), $75-80 \%$ were $<2 \mathrm{~cm}$, and $<10 \%$ received percutaneous needle biopsy preoperatively. However, this diagnostic approach led to $17 \%$ "unnecessary surgery" with an ultimately benign diagnosis. Correspondingly, recent reports have documented an accuracy of $85 \%$ of experienced radiologists to estimate the likelihood of malignancy of nodules $<2 \mathrm{~cm}$ based on CT-imaging, with or without additional help from risk prediction models (23). Although this seems to align with beforementioned guideline recommendations to accept upfront surgery when the clinical probability of malignancy is $>65-70 \%$ $(3,6)$, these rates of ultimately unnecessary surgery undeniably challenge the physician's ability to make a reliable lung cancer diagnosis solely based on clinical and radiologic data.

Secondly, the lack of a preoperative diagnosis requires the acquirement of an intraoperative diagnosis, usually through wedge resection and FS examination, before anatomic pulmonary resection can be justified (3). However, not all pulmonary nodules are suitable for this approach. In a recent Japanese case series with 199 patient undergoing lung resection without preoperative definitive diagnosis, 98 (49\%) underwent upfront direct lobectomy without FS, because wedge resection was deemed not feasible due to size and/or depth of the nodule (24); ultimately, six of these patients $(6 \%)$ had benign disease as final pathological diagnosis (24). Even when diagnostic wedge resection is feasible, various problems can arise hampering real-time intraoperative decision-making related with FS examination. Although the concordance rate between intraoperative FS 
results and final pathology diagnosis has been reported to be appropriate $(25,26)$, rates of false-negative and indeterminate FS diagnosis have been reported as high as $12-18 \%(27,28)$, and errors of intraoperative FS procedures are reported to have significant clinical impact (25). Furthermore, in the setting of a previous malignancy, the intraoperative differentiation between primary NSCLC or pulmonary metastasis might by problematic, while being essential in determining the extent of resection. Remarkably, the rate of IOD in our study was similar in patients with and without a history of previous cancer, suggesting that surgeons do not necessarily feel the need for this differentiation beforehand.

Thirdly, various additional challenges arise specifically in the setting of small-sized tumors while considering a potential sublobar resectional strategy when a preoperative definitive diagnosis is not available. Technically, a wedge resection necessary for confirming the cancer diagnosis might obscure intersegmental planes and complicate a following segmentectomy. Conversely, an upfront segmentectomy is technically more challenging than wedge resection, and is associated with higher surgical morbidity $(29,30)$ and mortality (31), exposing the patient to unnecessary surgical trauma and risks in the case of an ultimately benign diagnosis. Moreover, several adenocarcinoma subtypes [such as micropapillary adenocarcinoma (31)] and tumors with high-risk histologic features [such as "tumor spread through air space" (16)] are known to have an inferior prognosis after sublobar resection $(15,16,32)$, and lobectomy is therefore recommended. However, when a preoperative diagnosis is not available, the indispensable intraoperative FS examination has been associated with a diagnostic accuracy of only $68-85 \%$ in identifying predominant histologic subtype $(25,33)$. Additionally, various studies have documented low sensitivity for FS in diagnosing high-risk morphology, often caused by sampling error largely affected by tumor heterogeneity $(16,34)$. Consequently, errors in intraoperative FS examination are factual and well reported $(28,33)$, and could potentially have significant negative clinical impact (15). Therefore, an accurate preoperative pathological diagnosis with subtyping is essential to guide the surgeon in choosing the optimal intraoperative strategy regarding extent of resection, especially for smaller sized tumors and/or adenocarcinomas $(32,35)$. Interestingly, in our study, we observed an opposite clinical practice that lower T-stage, smaller sized tumors, adenocarcinoma, or undergoing segmentectomy were factors significantly associated with a higher IOD rate.
Naturally, the beforementioned drawbacks of IOD should be balanced against the hazards and accuracy of preoperative invasive diagnostic procedures. Traditionally, these nonsurgical biopsies can be obtained through guidance with bronchoscopy, ultrasound, computed tomography (CT), and more recently through electromagnetic navigation bronchoscopy (ENB) and cone-beam-CT based navigation bronchoscopy (3). For CT-guided transthoracic lung biopsy, major complication rate relatively high with a $19-25 \%$ risk of pneumothorax requiring drainage in $4.3-5.6 \%$ (36). The introduction of navigation bronchoscopy-guided biopsy is associated with a much lower risk of pneumothorax $(2-3 \%)$ (37). In our country however, navigation bronchoscopy is not widely implemented and is routinely used in our center alone since late 2017. Since the yield of image guided navigation bronchoscopy is increasing and in some centers matches the yield of TTNA, this is a promising strategy to implement $(38,39)$.

There were several important limitations to our current explorative study. First of all, the study was performed using data obtained from the NNCR, including only patients with a cancer diagnosis. With the absence of patients with benign pathology, we could not explore the effect of the lack of a preoperative diagnosis on the proportion of patients undergoing ultimately futile surgery, or properly assess any correlation with preoperative workup or postoperative outcome. In potential upcoming future studies on this matter, we plan to use data from population-based and nationwide surgical databases on general thoracic surgery to address the beforementioned issues.

The clinical implications of the high incidence of IOD and the remarkably large variation of this occurrence remain speculative at this moment. However, several prospects in impending trends in lung cancer practice should be considered in this regard. As advocated earlier, the negative effects of IOD would theoretically be most important in smaller-sized tumors, especially adenocarcinomas, particularly when considering a sublobar resectional strategy. With the advent of lung cancer screening with low-dose CT (40-42), a large number of patients with small pulmonary nodules will be detected and presented to multidisciplinary team meetings. In previous lung cancer screening trials, $49-81 \%$ of patients who will undergo surgical resection of these nodules do not have a preoperative diagnosis $(43,44)$. Furthermore, while $73-91 \%$ presented with clinical stage I disease, only $18-48 \%$ were treated by sublobar resections $(43,44)$. Comparably, the percentage of sublobar resections in our study is fairly low as well. Surgeons may not be familiar or comfortable yet 
performing sublobar resections, besides awaiting for more evidence supporting these resections. Although lobectomy has long been the standard resection for most patients with resectable lung cancer (45), randomized trials are currently assessing the results of sublobar resections for NSCLC $\leq 2 \mathrm{~cm}$ when compared to standard lobectomy $(12,46,47)$. The outcome of these trials could have significant impact on guidelines recommendations on the extent of surgical resection in these smaller sized tumors, especially with favorable histopathology, substantially increasing the number of patients in whom a sublobar resectional strategy could be considered. With the complex interplay between tumor size, pathology subtyping, and extent of resection on oncological outcome (48), the oncological and surgical communities should maximize efforts diminishing the current variations in care, unifying discordances in preoperative diagnostic algorithms, hopefully leading to more uniform perioperative decision-making regarding the extent of surgical resection, ultimately offering the best patient-tailored surgical care for individuals with NSCLC.

Another limitation of our study is the lack of data about the performed preoperative diagnostic workup. Unfortunately, these records were not included in the available NCCR dataset, and the exact utilization of invasive diagnostic procedures before surgery remains to be clarified. Notwithstanding this paucity of data, it is clear that the currently practiced diagnostic strategy only leads to a definitive preoperative diagnosis in only $64 \%$ of cases, with a wide variation of $33-87 \%$ between Dutch centers. This seems to be at variance with the recommendations by the Dutch guidelines (2), stating that "the diagnosis of nonsmall cell lung cancer is preferably obtained preoperatively", which is in line with other international documents on this matter, including the ESMO guidelines (4). However, other international standards advise the opposite, with the NCCN (5) recommending that "patients with a strong clinical suspicion of stage I or II lung cancer (based on risk factors and radiologic appearance) do not require a biopsy before surgery". In this regard, to diminish unwarranted variation in lung cancer care, international and national consensus should be advocated on this matter. As such, regional and national data, such as provided in our current study, should be examined as a benchmark for future reference.

\section{Conclusions}

In conclusion, our current study presents the first available nationwide population-based numbers on IOD rates in patients undergoing lung cancer surgery in the Netherlands, offering data for benchmarking purposes. The significant variation in these rates deserves additional attention in order to initiate uniform surgical quality of care for patients with lung cancer, especially when considering a sublobar resectional strategy.

\section{Acknowledgments}

Funding: None.

\section{Footnote}

Reporting Checklist: The authors have completed the STROBE reporting checklist. Available at https://dx.doi. org/10.21037/jtd-21-617

Peer Review File: Available at https://dx.doi.org/10.21037/ jtd-21-617

Conflicts of Interest: All authors have completed the ICMJE uniform disclosure form (available at https:// dx.doi.org/10.21037/jtd-21-617). van der Heijden EH and Verhagen AF report their department has received unrestricted research grants from Astra Zeneca, Pentax Medical and Philips Medical Systems, as well as speakers fees from Pentax Medical, out the submitted work. The other authors have no conflicts of interest to declare.

Ethical Statement: The authors are accountable for all aspects of the work in ensuring that questions related to the accuracy or integrity of any part of the work are appropriately investigated and resolved. The study was conducted in accordance with the Declaration of Helsinki (as revised in 2013). In accordance with the regulations of the Central Committee on Research involving Human Subjects, this type of study does not require approval from an ethics committee in the Netherlands.

Open Access Statement: This is an Open Access article distributed in accordance with the Creative Commons Attribution-NonCommercial-NoDerivs 4.0 International License (CC BY-NC-ND 4.0), which permits the noncommercial replication and distribution of the article with the strict proviso that no changes or edits are made and the original work is properly cited (including links to both the formal publication through the relevant DOI and the license). See: https://creativecommons.org/licenses/by-nc-nd/4.0/. 


\section{References}

1. Howington JA, Blum MG, Chang AC, et al. Treatment of Stage I and II Non-Small Cell Lung Cancer: Diagnosis and Management of Lung Cancer, 3rd Ed: American College of Chest Physicians Evidence-Based Clinical Practice Guidelines. Chest 2013;143:e278S-e313S.

2. Oncoline. The Dutch Clinical Practice Guidelines on NSCLC (2.3). Available online: https://www.oncoline.nl/ niet-kleincellig-longcarcinoom

3. Callister ME, Baldwin DR, Akram AR, et al. British Thoracic Society guidelines for the investigation and management of pulmonary nodules. Thorax 2015;70 Suppl 2:ii1-ii54.

4. Postmus PE, Kerr KM, Oudkerk M, et al. Early and locally advanced non-small-cell lung cancer (NSCLC): ESMO Clinical Practice Guidelines for diagnosis, treatment and follow-up. Ann Oncol 2017;28:iv1-iv21.

5. Ettinger et al. National Comprehensive Cancer Network Clinical Practice Guidelines in Oncology - Non-Small Cell Lung Cancer version 3.2020 - February 11, 2020 [cited $2021 \mathrm{Feb} 5$ ]. Available online: https://www2.trikobe.org/nccn/guideline/lung/english/non_small.pdf

6. Gould MK, Donington J, Lynch WR, et al. Evaluation of individuals with pulmonary nodules: when is it lung cancer? Diagnosis and management of lung cancer, 3rd ed: American College of Chest Physicians evidence-based clinical practice guidelines. Chest 2013;143:e93S-e120S.

7. Heo EY, Lee KW, Jheon S, et al. Surgical resection of highly suspicious pulmonary nodules without a tissue diagnosis. Jpn J Clin Oncol 2011;41:1017-22.

8. Kuo E, Bharat A, Bontumasi N, et al. Impact of videoassisted thoracoscopic surgery on benign resections for solitary pulmonary nodules. Ann Thorac Surg 2012;93:266-72; discussion 272-3.

9. Sihoe AD, Hiranandani R, Wong H, et al. Operating on a suspicious lung mass without a preoperative tissue diagnosis: pros and cons. Eur J Cardiothorac Surg 2013;44:231-7; discussion 237.

10. Ozeki N, Iwano S, Taniguchi T, et al. Therapeutic surgery without a definitive diagnosis can be an option in selected patients with suspected lung cancer. Interact Cardiovasc Thorac Surg 2014;19:830-7.

11. Cho J, Ko SJ, Kim SJ, et al. Surgical resection of nodular ground-glass opacities without percutaneous needle aspiration or biopsy. BMC Cancer 2014;14:838.

12. Kohman LJ, Gu L, Altorki N, et al. Biopsy first: Lessons learned from Cancer and Leukemia Group B (CALGB) 140503. J Thorac Cardiovasc Surg 2017;153:1592-7.

13. Madariaga ML, Lennes IT, Best T, et al. Multidisciplinary selection of pulmonary nodules for surgical resection: Diagnostic results and long-term outcomes. J Thorac Cardiovasc Surg 2020;159:1558-1566.e3.

14. McMurry TL, Shah PM, Samson P, et al. Treatment of stage I non-small cell lung cancer: What's trending? J Thorac Cardiovasc Surg 2017;154:1080-7.

15. Beasley MB, Dembitzer FR, Flores RM. Surgical pathology of early stage non-small cell lung carcinoma. Ann Transl Med 2016;4:238.

16. Takahashi Y, Kuroda H, Oya Y, et al. Challenges for realtime intraoperative diagnosis of high risk histology in lung adenocarcinoma: A necessity for sublobar resection. Thorac Cancer 2019;10:1663-8.

17. Engelhardt KE, Feinglass JM, DeCamp MM, et al. Treatment trends in early-stage lung cancer in the United States, 2004 to 2013: A time-trend analysis of the National Cancer Data Base. J Thorac Cardiovasc Surg 2018;156:1233-1246.e1.

18. Rich AL, Baldwin DR, Beckett P, et al. ERS statement on harmonised standards for lung cancer registration and lung cancer services in Europe. Eur Respir J 2018;52:1800610.

19. Clark JM, Marrufo AS, Kozower BD, et al. Cardiopulmonary Testing Before Lung Resection: What Are Thoracic Surgeons Doing? Ann Thorac Surg 2019;108:1006-12.

20. Sineshaw HM, Sahar L, Osarogiagbon RU, et al. CountyLevel Variations in Receipt of Surgery for Early-Stage Non-small Cell Lung Cancer in the United States. Chest 2020;157:212-22.

21. Khakwani A, Rich AL, Tata LJ, et al. The pathological confirmation rate of lung cancer in England using the NLCA database. Lung Cancer 2013;79:125-31.

22. Izumo T, Terada Y, Inomata $M$, et al. Impact of preoperative pathological confirmation on surgical and postoperative outcomes of lung resection for early stage lung cancer. Adv Respir Med 2019;87:203-8.

23. MacMahon H, Li F, Jiang Y, et al. Accuracy of the Vancouver Lung Cancer Risk Prediction Model Compared With That of Radiologists. Chest 2019;156:112-9.

24. Mori S, Noda Y, Shibazaki T, et al. Definitive lobectomy without frozen section analysis is a treatment option for large or deep nodules selected carefully with clinical diagnosis of malignancy. Thorac Cancer 2020;11:1996-2004.

25. Liu S, Wang R, Zhang Y, et al. Precise Diagnosis of Intraoperative Frozen Section Is an Effective Method to Guide Resection Strategy for Peripheral Small-Sized Lung Adenocarcinoma. J Clin Oncol 2016;34:307-13.

26. Zhao ZR, Lau RWH, Long H, et al. Novel method for rapid identification of micropapillary or solid components in early-stage lung adenocarcinoma. J Thorac Cardiovasc 
Surg 2018;156:2310-2318.e2.

27. Marchevsky AM, Changsri C, Gupta I, et al. Frozen section diagnoses of small pulmonary nodules: accuracy and clinical implications. Ann Thorac Surg 2004;78:1755-9.

28. Walts AE, Marchevsky AM. Root cause analysis of problems in the frozen section diagnosis of in situ, minimally invasive, and invasive adenocarcinoma of the lung. Arch Pathol Lab Med 2012;136:1515-21.

29. Altorki NK, Kamel MK, Narula N, et al. Anatomical Segmentectomy and Wedge Resections Are Associated with Comparable Outcomes for Patients with Small cT1N0 NonSmall Cell Lung Cancer. J Thorac Oncol 2016;11:1984-92.

30. Tuminello S, Wolf A, Taioli E, et al. Comparison of Wedge Versus Lobar Resection for Stage 1 Non-Small Cell Lung Cancer: A SEER-Medicare Analysis. Ann Thorac Surg 2018;106:1260-1.

31. Seder CW, Wright CD, Chang AC, et al. The Society of Thoracic Surgeons General Thoracic Surgery Database Update on Outcomes and Quality. Ann Thorac Surg 2016;101:1646-54.

32. Zugazagoitia J, Enguita AB, Nuñez JA, et al. The new IASLC/ATS/ERS lung adenocarcinoma classification from a clinical perspective: current concepts and future prospects. J Thorac Dis 2014;6:S526-36.

33. Yeh YC, Nitadori J, Kadota K, et al. Using frozen section to identify histological patterns in stage I lung adenocarcinoma of $\leq 3 \mathrm{~cm}$ : accuracy and interobserver agreement. Histopathology 2015;66:922-38.

34. Aliyev N, Takahashi Y, Lu S, et al. Intraoperative subtyping of lung adenocarcinoma: an unmet need. Transl Cancer Res 2016;5:S40-3.

35. Travis WD, Brambilla E, Noguchi M, et al. International association for the study of lung cancer/american thoracic society/european respiratory society international multidisciplinary classification of lung adenocarcinoma. J Thorac Oncol 2011;6:244-85.

36. Heerink WJ, de Bock GH, de Jonge GJ, et al. Complication rates of CT-guided transthoracic lung biopsy: meta-analysis. Eur Radiol 2017;27:138-48.

37. Folch EE, Labarca G, Ospina-Delgado D, et al. Sensitivity and Safety of Electromagnetic Navigation Bronchoscopy for Lung Cancer Diagnosis: Systematic Review and Metaanalysis. Chest 2020;158:1753-69.

38. Verhoeven RLJ, Fütterer JJ, Hoefsloot W, et al. Cone-Beam CT Image Guidance With and Without Electromagnetic Navigation Bronchoscopy for Biopsy of Peripheral Pulmonary Lesions. J Bronchology Interv Pulmonol 2021;28:60-9.

39. Pritchett MA, Bhadra K, Mattingley JS. Electromagnetic
Navigation Bronchoscopy With Tomosynthesisbased Visualization and Positional Correction: Threedimensional Accuracy as Confirmed by Cone-Beam Computed Tomography. J Bronchology Interv Pulmonol 2021;28:10-20.

40. Veronesi G, Baldwin DR, Henschke CI, et al. Recommendations for Implementing Lung Cancer Screening with Low-Dose Computed Tomography in Europe. Cancers (Basel) 2020;12:1672.

41. National Lung Screening Trial Research Team; Aberle DR, Adams AM, et al. Reduced lung-cancer mortality with low-dose computed tomographic screening. N Engl J Med 2011;365:395-409.

42. de Koning HJ, van der Aalst CM, de Jong PA, et al. Reduced Lung-Cancer Mortality with Volume CT Screening in a Randomized Trial. N Engl J Med 2020;382:503-13.

43. Flores R, Bauer T, Aye R, et al. Balancing curability and unnecessary surgery in the context of computed tomography screening for lung cancer. J Thorac Cardiovasc Surg 2014;147:1619-26.

44. Ho H, Williamson C, Regis SM, et al. Surgery and invasive diagnostic procedures for benign disease are rare in a large low-dose computed tomography lung cancer screening program. J Thorac Cardiovasc Surg 2021;161:790-802.e2.

45. Ginsberg RJ, Rubinstein LV. Randomized trial of lobectomy versus limited resection for T1 N0 non-small cell lung cancer. Lung Cancer Study Group. Ann Thorac Surg 1995;60:615-22; discussion 622-3.

46. Nakamura K, Saji H, Nakajima R, et al. A phase III randomized trial of lobectomy versus limited resection for small-sized peripheral non-small cell lung cancer (JCOG0802/ WJOG4607L). Jpn J Clin Oncol 2010;40:271-4.

47. Yang F, Sui $X$, Chen $X$, et al. Sublobar resection versus lobectomy in Surgical Treatment of Elderly Patients with early-stage non-small cell lung cancer (STEPS): study protocol for a randomized controlled trial. Trials 2016;17:191.

48. Raman V, Jawitz OK, Voigt SL, et al. The Effect of Tumor Size and Histologic Findings on Outcomes After Segmentectomy vs Lobectomy for Clinically Node-Negative Non-Small Cell Lung Cancer. Chest 2021;159:390-400.

Cite this article as: Ghamati MR, Li WWL, van der Heijden EHFM, Verhagen AFTM, Damhuis RA. Surgery without preoperative histological confirmation of lung cancer: what is the current clinical practice? J Thorac Dis 2021;13(10):57655775. doi: 10.21037/jtd-21-617 